\title{
Pharmacogenomics and personalized medicine: a good help towards innovative chemotherapeutic strategies?
}

\section{Editorial}

Personalized medicine is often based on the ethical use of individual's genetic profile, and contributes to make the best theranostic option. Pharmacogenomics is a genomic approach that consists to study the individual response to drugs (e.g. semi-synthetic, synthetic or natural compounds). Adverse drug reactions are a common and important complication worldwide. Although, the relative risk/benefit ratio of approved drugs (e.g. by US Food and Drug Administration (FDA), European Agency of Drugs (EMEA)) is generally low, a non-negligible percentage of marketed drugs, including the original, generic, sub-standard and counterfeited ones, induce millions of annual deaths worldwide (i.e. the so-called "iatrogenic effects").

Over the past decade, thanks to the last generation genomic platforms (e.g. microarrays, next-generation sequencing (NGS)) and the completion of the Human Genome Project, it has become increasingly apparent that submicroscopic genomic alterations (SNPs, CNVs) are important determinants of drug-resistance and drug-related adverse events, strongly suggesting that genetic variants (e.g. epigenetic modifiers, polymorphisms) can be reliable predictive biomarkers of drug resistance and drug-associated complications. Genomic variants are known to be involved in a number of pathologies and cellular processes (e.g. regulation of gene expression such as $\mathrm{ABC}$ transporters, regulation of pharmacokinetics (i.e. absorption, distribution, metabolism and excretion - ADME) and pharmacodynamics (i.e. molecular action of a drug on its target). It is clear that it would be too difficult to design a specific drug for each patient. Therefore, the rational design of drugs by pharmaceutical companies shall consider the most common pharmacogenomics profile of a given large population. Furthermore, healthcare professionals shall routinely and ethically use the individual's genetic profile to choose the best theranostic strategy. These recommendations should lead in better changes in public policy and clinical practice to minimize errors in disease diagnosis as well as enhance drug efficacy and safety in a given patient (e.g. increase of disease targeting, decrease of side
Volume I Issue 2 - 2014

\section{Farid Menaa}

Department of Oncology, Stem cells and Nanomedicine, Fluorotronics Inc., USA

Correspondence: Farid Menaa, Department of Oncology, Stem cells and Nanomedicine, Fluorotronics Inc., 2453 Cades Way,Vista, CA 9208I, USA, Email dr.fmenaa@gmail.com

Received: June 16,2014 | Published: June 19, 2014

effects such as drug-resistance and those related to toxic doses to obtain an expected effect).

Taken together, the evidence-based personalized medicine recognizes that no two patients are alike, and subsequent clinical decisions shall contribute to adjust the drug dosage and choose the best drug. However, in some diseases, such as cancers, the search for further determinants such as initiating/propagating cells, which may contribute to poor prognosis and drug resistance (e.g. over-expression of $\mathrm{ABC}$ transporters at their surface to efflux drugs), are necessary. Eventually, one should keep in mind that a negative genome-based diagnosis result does not necessarily means a good predictive result, and a holistic medical approach (i.e. based on multi- and interdisciplines) is definitely an asset for obtaining a reliable "big picture" of what happen and what would happen.

\section{Acknowledgements}

None.

\section{Conflict of interest}

Author declares that there is no conflict of interest. 